\title{
Spontaneous Formation of Dipolar Metal Nanoclusters
}

\author{
Elizabeth A. Sokol, Sara E. Mason, Valentino R. Cooper and Andrew M. Rappe \\ The Makineni Theoretical Laboratories, Department of Chemistry \\ University of Pennsylvania, Philadelphia, PA 19104-6323
}

(Dated: October 5, 2018)

\begin{abstract}
The adsorption of $\mathrm{Ag}_{3}$ and $\mathrm{Ag}_{4}$ clusters on the $\alpha-\mathrm{Al}_{2} \mathrm{O}_{3}(0001)$ surface is explored with density functional theory. Within each adsorbed cluster, two different cluster-surface interactions are present. We find that silver clusters simultaneously form both ionic bonds with surface oxygen and intermetallic bonds with surface aluminum. The simultaneous formation of disparate electronic structure motifs within a single metal nanoparticle is termed a "dipolar nanocluster". This coexistence is ascribed to the similar bond enthalpies of $\mathrm{Ag}-\mathrm{Al}$ and $\mathrm{Ag}-\mathrm{O}$ bonds, and its importance for nanoparticle catalysis is highlighted.
\end{abstract}

There is great fundamental interest in understanding how transition metals and oxides are affected by contact with each other. Since transition metal/oxide interfaces exert significant controllable influence on material properties, many current and potential applications rely on these heterostructures, including catalysts for automotive pollution control 1] and fuel cells 2], as well as nanoscale biosensors. 3] Recent theoretical [4, 5, 6] and experimental [7, 8, 9] studies of size-selected nanocluster deposition onto oxides highlight size dependence of the chemical and physical properties. Furthermore, numerous experimental and theoretical investigations emphasize the role of the oxide support in changing the catalytic ability of these metal-oxide systems. 10, 11, 12, 13, 14, 15, 16, 17.

The combination of $\mathrm{Ag}$ and $\mathrm{Al}_{2} \mathrm{O}_{3}$ is special, because of the close competition between ionic $(\mathrm{Ag}-\mathrm{O})$ and intermetallic $(\mathrm{Ag}-\mathrm{Al})$ bonding. $\mathrm{Ag}$ and $\mathrm{Au}$ have similarly low oxide formation energies. 18 However, Au bonds to $\mathrm{Al}$ much more strongly than to $\mathrm{O}$, whereas the bonds that $\mathrm{Ag}$ makes to $\mathrm{Al}$ and to $\mathrm{O}(\mathrm{Ag}-\mathrm{Al} \approx 1.91 \mathrm{eV}$ and $\mathrm{Ag}-\mathrm{O} \approx 2.28 \mathrm{eV})$ are more similar than any other element (only $\mathrm{Cu}$ is close). 19]

In the present Letter, we report a novel consequence of this bonding competition: small supported $\mathrm{Ag}_{n}$ clusters exhibit two coexisting structural and electronic relationships to an $\alpha-\mathrm{Al}_{2} \mathrm{O}_{3}(0001)$ ( $\alpha$-alumina) substrate. The proximity of such different states within one cluster is fundamentally interesting, and has ramifications for understanding and improving noble metal nanocluster catalysis. We use first-principles density functional theory (DFT) to study the bonding of three- and fouratom $\mathrm{Ag}$ clusters to the Al-terminated (0001) surface of $\alpha-\mathrm{Al}_{2} \mathrm{O}_{3}$. We find that bonding competition strongly influences the stable cluster adsorption geometries, and is directly responsible for inducing unusual electronic states in these clusters.

DFT calculations were performed with a generalizedgradient approximation exchange-correlation functional. [20] Geometry optimizations were carried out using an in-house code, and calculation of Born effective charges [21] and orbital-projected density of states (PDOS) were done using the ABINIT software package. 22] All calculations were converged using a $2 \times 2 \times 1$ grid of Monkhorst-Pack $k$-points. 23] Norm-conserving optimized pseudopotentials 24] with the designed nonlocal method for metals [25, 26] were constructed using the OPIUM pseudopotential package. 27] The Kohn-Sham orbitals are expanded in a plane-wave basis set truncated at $50 \mathrm{Ry}$.

The $\alpha-\mathrm{Al}_{2} \mathrm{O}_{3}$ surface is modeled by a slab geometry supercell with an in-plane $(\sqrt{3} \times \sqrt{3}) R 30^{\circ}$ unit cell and periodic boundary conditions. The slabs consist of five $\mathrm{Al}_{3} \mathrm{O}_{9} \mathrm{Al}_{3}$ tri-layers, making the surfaces Al-terminated. At least $12 \AA$ of vacuum separate periodic images in the (0001) direction. The theoretical $\mathrm{Al}_{2} \mathrm{O}_{3}$ in-plane lattice constant of $4.798 \AA$ was used (4.759 $\AA$ experimental [28]).

At equilibrium, the surface $\mathrm{Al}$ layer is only $z \approx 0.1 \AA$ above the $\mathrm{O}$ layer. In-plane relaxation yields nearestneighbor O-O distances from $2.63 \AA$ to $2.94 \AA \AA$. Structural details of the alumina surface are consistent with other modeling studies 29].

Chemisorption of $\mathrm{Ag}$ on the $\alpha-\mathrm{Al}_{2} \mathrm{O}_{3}$ (0001) surface strongly favors cluster formation 30, 31] because of 4 $5 \%$ mismatch between the surface $\mathrm{O}-\mathrm{O}$ distance $(2.76 \AA)$ and the $\mathrm{Ag}-\mathrm{Ag}$ distance $(2.88 \AA)$ in bulk $\mathrm{Ag}$. To find the minimum-energy structures for $\mathrm{Ag}_{3}$, for planar $\mathrm{Ag}_{4}$, and for pyramidal $\mathrm{Ag}_{4}$, each cluster was initially placed three different ways. Interfacial $\mathrm{Ag}$ atoms were started at top, bridge, or hollow sites of the oxygen lattice, and each system was allowed to relax fully. In each calculation, the top two surface tri-layers of alumina were allowed to relax in all directions, the third tri-layer was relaxed in the direction normal to the surface plane, and the bottom two tri-layers were constrained to their bulk alumina coordinates.

For all starting positions, geometry optimization of $\mathrm{Ag}_{3} / \mathrm{Al}_{2} \mathrm{O}_{3}$ leads to the same final structure (Figure 1), with all $\mathrm{Ag}$ atoms in hollow sites of the top O layer lattice, one above a surface $\mathrm{Al}$ atom, and two above subsurface $\mathrm{Al}$ atoms. The cluster chemisorption energy $E_{\text {ads }}$ is $2.09 \mathrm{eV}$. The cluster tilts by $30^{\circ}$ with respect to the surface plane, with the $\mathrm{Ag}$ above the surface $\mathrm{Al}$ farthest from the surface. Defining $z$ as the distance of $\mathrm{Al}$ atoms from the topmost oxygen layer in the surface normal direction, the values in bare $\mathrm{Al}_{2} \mathrm{O}_{3}$ are $0.096 \AA$ for the topmost $\mathrm{Al}$ of the unit cell. In response to cluster adsorption, the nearest surface $\mathrm{Al}$ atoms move vertically by $\Delta z=-0.47$, - 


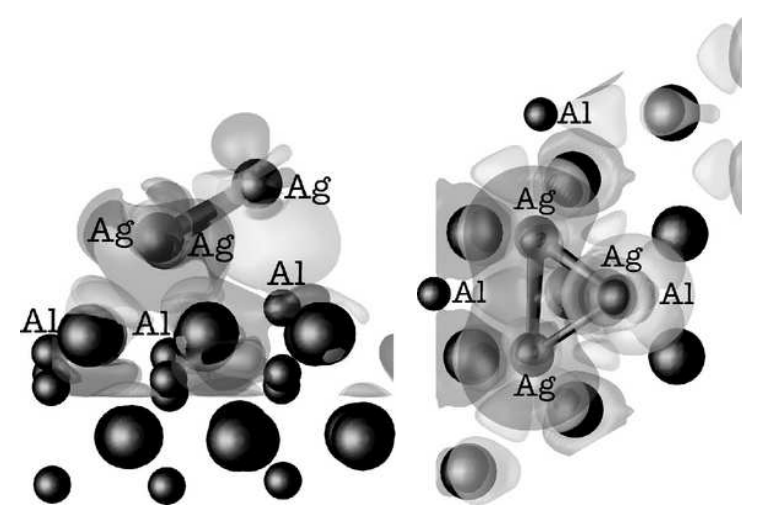

(a)

(b)

FIG. 1: Induced charge density $\Delta \rho$ diagrams for the optimized structure of $\mathrm{Ag}_{3} / \mathrm{Al}_{2} \mathrm{O}_{3}$. O, $\mathrm{Ag}$ (connected), and $\mathrm{Al}$ are shown as black spheres of decreasing size. Adsorption causes electron flow from dark to light regions. Topmost $\mathrm{Ag}$ and $\mathrm{Al}$ atoms are labeled. Iso-surface values are $\pm 0.02 e^{-} / \AA^{3}$. (a) Side view. (b) Top view.

0.55, and $+0.57 \AA$. One $\mathrm{Ag}$ and one $\mathrm{Al}$ atom shift upward together. The inward relaxation of the the other nearby surface $\mathrm{Al}$ atoms induced by metal cluster adsorption has been observed in other theoretical studies 32].

The relaxed interatomic distances strongly suggest two different bonding motifs for the $\mathrm{Ag}_{3}$ cluster. The raised $\mathrm{Ag}$ and $\mathrm{Al}$ make a short $2.65 \AA$ bond, which is just about the sum of their covalent radii $\left(r_{\mathrm{Ag}}=1.53 \AA, r_{\mathrm{Al}}=1.18 \AA\right)$. The shortest distance from this $\mathrm{Ag}$ to $\mathrm{O}$ is $3.66 \AA$, much longer than the sum of their ionic radii $\left(r_{\mathrm{Ag}^{+}}=1.14 \AA\right.$, $\left.r_{\mathrm{O}^{2-}}=1.24 \AA\right)$. The other two $\mathrm{Ag}$ atoms have short $\approx 2.46 \AA$ distances to $\mathrm{O}$, quite in line with ionic bonding. These Ag atoms also have long distances of $2.95 \AA$ to subsurface Al, suggesting little if any covalent interaction in that case. So the bond length data can be summarized as a covalent (intermetallic, IM) bond between the raised $\mathrm{Ag}$ and $\mathrm{Al}$, and ionic $\mathrm{Ag}-\mathrm{O}$ bonds for the other two $\mathrm{Ag}$ atoms.

We study $\Delta \rho$, the change in charge density induced by the cluster adsorption, to visualize the electronic clustersurface interactions. The side and top views of the isosurfaces for $\Delta \rho$ are shown in Figure 11 with electronic charge flowing from dark to light regions. Figure 1 shows gain of charge between the raised $\mathrm{Ag}$ and $\mathrm{Al}$, indicative of $\mathrm{Ag}-\mathrm{Al} \mathrm{IM}$ bond formation. The other two Ag atoms show a significant loss of electrons, with a corresponding gain of electrons for the nearest topmost surface oxygens. Therefore, these interactions are chiefly ionic bonds.

This electronic description of $\mathrm{Ag}_{3}$ adsorption also provides insight into the observed surface relaxation. The ionic bonds formed between $\mathrm{Ag}$ and $\mathrm{O}$ leave these $\mathrm{O}$ atoms less capable of bonding to surface $\mathrm{Al}$ atoms, using a bond-valence argument. 33. Therefore, these adjacent surface Al atoms relax inward (below the top O layer),
TABLE I: Principal values of Born effective charge tensors and electronic $d$-band centers $\epsilon_{d}$ for supported cluster $\mathrm{Ag}$ atoms. For each atom, the largest principal value of each sign, along with the corresponding principal direction, are reported. The principal directions (shown in Figure 3) in all cases point inward toward the cluster center and along the surface normal. The angle $\theta$ between the principal direction and the surface normal are tabulated.

\begin{tabular}{lccc} 
& Principal Value & $\theta{ }^{\circ}$ & $\epsilon_{d}, \mathrm{eV}$ \\
\hline $\mathrm{Ag}_{3}$ Ionic & 1.11 & 32 & -3.42 \\
$\mathrm{Ag}_{3}$ IM & -0.23 & 60 & -4.32 \\
Boat ionic & 1.45 & 31 & -3.14 \\
Boat IM & -0.42 & 41 & -3.66 \\
Boat IM & 0.11 & 87 & -3.66 \\
Candlestick ionic & 1.22 & 26 & -3.63 \\
Candlestick IM & -0.35 & 59 & -4.56 \\
Pyr ionic & 1.48 & 23 & -3.39 \\
Pyr IM & -0.27 & 0 & -4.16 \\
Pyr IM & 0.36 & 85 & -4.16 \\
Top Ag & -0.11 & isotropic & -3.78
\end{tabular}

so they can form bonds with subsurface oxygen. The increase in electron density near the IM Al reduces its electrostatic interaction with oxygen and results in the observed outward relaxation.

We propose referring to these clusters as "(electric) dipolar nanoparticles." (Magnetic) dipolar nanoparticles have been reported [34], but we know of no previous report showing spontaneous formation of electric dipoles on nano-sized supported metal particles.

To make precise the magnitude and orientation of the nanoparticle dipole, we apply the modern theory of polarization. 21] The Born effective charge tensor $Z^{*}$ is found [35, 36], where the tensor element $Z_{\alpha i j}^{*}$ gives the change in electric polarization component $P_{i}$ as atom $\alpha$ moves along direction $j$. There is no requirement that this mixed second derivative tensor be symmetric, and the tensors of the supported clusters are highly anisotropic. We have obtained the principal values of the charge tensors and for each supported Ag atom the largest principal values (of each sign, where applicable) and their corresponding principal directions are reported in Table I

The dynamical charge values support the above interpretation of the $\mathrm{Ag} / \mathrm{Al}_{2} \mathrm{O}_{3}$ interactions: ionically bound $\mathrm{Ag}$ atoms lose charge through interaction with surface $\mathrm{O}$, resulting in positive principal values. The IM Ag atom gains charge from the bonding with surface $\mathrm{Al}$, resulting in a modest negative $Z^{*}$, but most of the charge is shared, not closely associated with $\mathrm{Ag}$ motion. In some cases (boat, pyr) the IM atoms have a more complicated Born effective charge tensor, with positive and negative principal values. The principal directions all point inward to the center of the cluster and out of the surface plane (Figure 3). Increased negative charge on the IM Al atom and ionic $\mathrm{O}$ atoms in the adsorbed geometry relative to the bare surface compensate for the net positive charge localized on the cluster. 


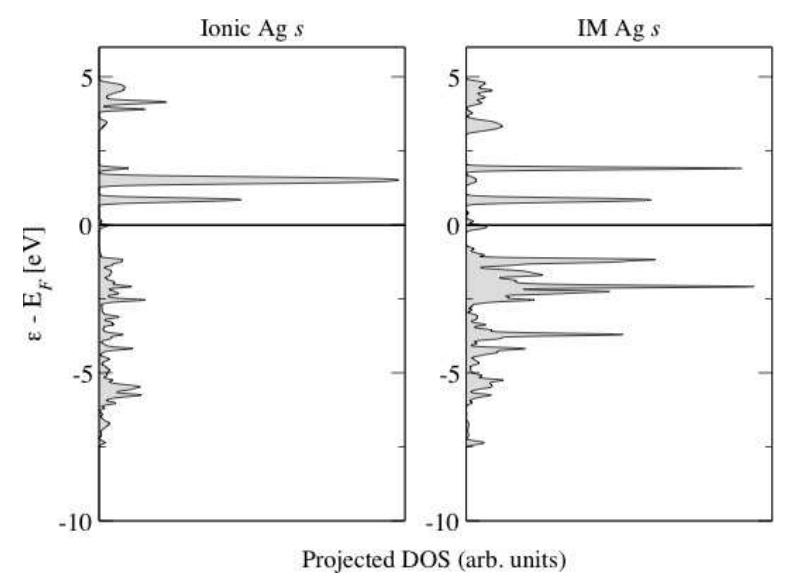

FIG. 2: Density of states projected onto the $s$-orbital of $\mathrm{Ag}$ atoms in $\mathrm{Ag}_{3} / \mathrm{Al}_{2} \mathrm{O}_{3}$.

Ionic and IM Ag-surface interactions are also revealed in PDOS analysis. Covalent IM bonding causes orbitals to mix, leading to intensity in the IM Ag $s$-PDOS below the Fermi level (Figure 2). The $s$ orbital of the ionic Ag shows less intensity at bonding levels, and is dominated by substantial intensity above the Fermi level. The fractional fillings of the IM and ionic Ag $s$ orbitals are 0.69 and 0.35 , respectively.

The projection onto $\mathrm{Ag} d$-states furhter demonstrates how the coexistence of two cluster/surface interactions affects the reactivity of the supported $\mathrm{Ag}$ atoms. Our analysis shows that the filling of the supported $\mathrm{Ag} d$ bands is constant and near unity for all Ag atoms. Table【 lists the average energy of the $d$-band projections $\left(\epsilon_{d}\right)$ of supported Ag atoms with respect to the Fermi level. $\epsilon_{d}$ is well established as a predictive parameter for assessing reactivity [37]. The $0.90 \mathrm{eV}$ shift in $\epsilon_{d}$ between the ionic and IM Ag atoms in $\mathrm{Ag}_{3} / \mathrm{Al}_{2} \mathrm{O}_{3}$ is larger then what can be achieved through perturbations such as strain 38] and is more in line with the extent of shift brought about by significant coordination change [39] or introduction of a metal hetero metal atom to a surface [40].

Having built a model of the chemisorption bonding in the $\mathrm{Ag}_{3} / \mathrm{Al}_{2} \mathrm{O}_{3}$ system, we examine how the chemisorption of $\mathrm{Ag}_{4}$ onto $\mathrm{Al}_{2} \mathrm{O}_{3}$ expands these ideas. As with the $\mathrm{Ag}_{3} / \mathrm{Al}_{2} \mathrm{O}_{3}$ system, calculations of $Z^{*}$ and PDOS were carried out on all optimized cluster geometries, and key results are discussed.

Pyramidal $\mathrm{Ag}_{4}$ ("pyr") bonds to $\mathrm{Al}_{2} \mathrm{O}_{3}$ $\left(E_{\text {ads }}=1.96 \mathrm{eV}\right)$ such that the three base atoms tilt and interact with the surface very similarly to $\mathrm{Ag}_{3} / \mathrm{Al}_{2} \mathrm{O}_{3}$. This and near-zero principal values of $Z^{*}$ for the pyramidal top $\mathrm{Ag}$ atom imply that the base screens the top atom from electrostatic interaction with the surface, consistent with previous results concerning the length scale of metal-oxide interactions. 41] A single $\mathrm{Ag}-\mathrm{Al} \mathrm{IM}$ bond is formed, and the other two Ag atoms participate in ionic interactions with the topmost surface oxygen. The $\mathrm{Al} \Delta z$ values are similar to those found

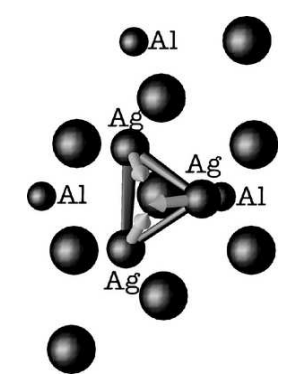

(a)

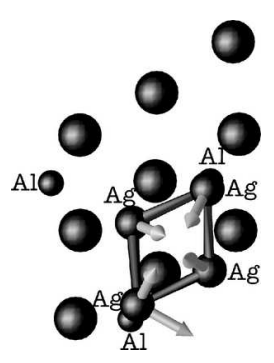

(c)

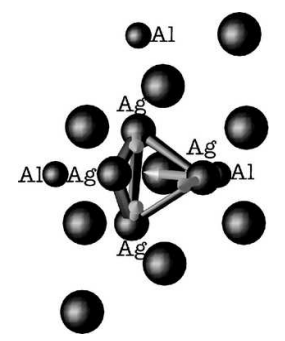

(b)

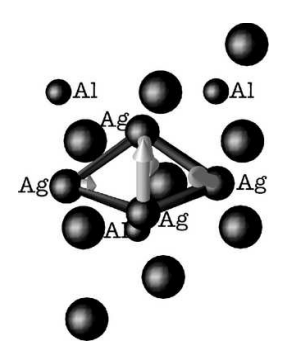

(d)
FIG. 3: Top views of optimized cluster geometries. $\mathrm{O}, \mathrm{Ag}$ (connected), and $\mathrm{Al}$ are shown as black spheres of decreasing size. Unit vectors of the principal directions of the Born effective charge principal values are indicated by arrows. Only the topmost nine $\mathrm{O}$ and three $\mathrm{Al}$ surface atoms of the unit cell are shown. (a) $\mathrm{Ag}_{3}$ (b) Pyr (c) Boat (d) Candlestick

in the $\mathrm{Ag}_{3} / \mathrm{Al}_{2} \mathrm{O}_{3}$ structure. The top $\mathrm{Ag}$ atom of the pyramid in the supported geometry is not in the $\mathrm{Ag}$ trimer hollow, but is shifted almost to the bridge site, above the two charge-depleted ionic Ag atoms.

The bonding competition between $\mathrm{Ag}-\mathrm{Al}$ and $\mathrm{Ag}-\mathrm{O}$ directly leads to two metastable minima for the planar $\mathrm{Ag}_{4} / \mathrm{Al}_{2} \mathrm{O}_{3}$ system. Each starting structure is a parallelogram parallel to the surface. The cluster buckles significantly as it chemisorbs. We find two local minima, each with all $\mathrm{Ag}$ atoms in hollow sites of the $\mathrm{O}$ lattice; the structures differ in their registry with the top $\mathrm{Al}$ sublattice. The ground state ("boat") has two Ag atoms above surface $\mathrm{Al}$, while the other local minimum ("candlestick") has only one $\mathrm{Ag}-\mathrm{Al}$ interaction.

The boat and candlestick $\mathrm{Ag}_{4}$ clusters have $E_{\text {ads }}$ values of $2.14 \mathrm{eV}$ and $1.49 \mathrm{eV}$, respectively. The boat is energetically favored, suggesting that a balance of IM and ionic bonding stabilized both. In fact, the boat exhibits larger positive and negative $Z^{*}$ values than the candlestick. Therefore, the energetically favored $\mathrm{Ag}_{4}$ boat cluster will exhibit even stronger dipolar nanoparticle properties than $\mathrm{Ag}_{3}$.

In conclusion, we find that bonding competition between $\mathrm{Ag}-\mathrm{Al}$ and $\mathrm{Ag}-\mathrm{O}$ gives rise to ionic $\mathrm{Ag}-\mathrm{O}$ and intermetallic $\mathrm{Ag}-\mathrm{Al}$ interactions between $\mathrm{Ag}$ cluster atoms 
and the alumina surface. The proximal coexistence of these interactions results in the formation of dipolar nanoparticles. The electronic and structural effects are closely related, with IM and ionic Ag-surface bonding favoring outward and inward $\mathrm{Al}$ motion, respectively. We find consistent results and interpretations of induced charge density, Born effective charges, and projected density of states in all four optimized cluster geometries $\left(\mathrm{Ag}_{3}\right.$, pyr, boat, and candlestick). Principal values of $Z^{*}$ show that ionic Ag atoms with positive charge and IM atoms with negative charge can be clearly distinguished, while stacked Ag atoms are mostly screened. The coexis- tence of ionic and IM bonding in supported clusters may be possible to create in other metal-oxide combinations, where the oxide and IM bond enthalpies are inherently similar or by tuning the competition by means of surface modification.

This work was supported by the Air Force Office of Scientific Research, Air Force Materiel Command, USAF, under Grant No. FA9550-04-1-0077. Computational support was provided by the Defense University Research Instrumentation Program, and the HighPerformance Computing Modernization Program of the U. S. Department of Defense.
[1] B. C. Gates, Chem. Rev. 95, 511 (1995).

[2] J. P. Breen, R. Burch, and H. M. Coleman, Appl. Catal., B 39, 65 (2002).

[3] A. V. Whitney, J. W. Elam, S. L. Zou, A. V. Zinovev, P. C. Stair, G. C. Schatz, and R. P. Van Duyne, J. Phys. Cehm. B 109, 20522 (2005).

[4] A. Eichler, Phys. Rev. B 68, 205408(1) (2003).

[5] A. Asthagiri and D. S. Sholl, Phys. Rev. B 73, 125432(1) (2006).

[6] M. W. Finnis, J. Phys.: Condens. Matter 8, 5811 (1996).

[7] A. Sanchez, S. Abbet, U. Heiz, W. D. Schneider, H. Hakkinen, R. N. Barnett, and U. Landman, J. Phys. Chem. A 103, 9573 (1999).

[8] S. Lee, C. Y. Fan, T. P. Wu, and S. L. Anderson, J. Amer. Chem. Soc. 126, 5682 (2004).

[9] L. Benz, X. Tong, P. Kemper, Y. Lilach, A. Kolmakov, H. Metiu, M. T. Bowers, and S. K. Buratto, J. Chem. Phys. 122, 0811021 (2005).

[10] S. Roberts and R. J. Gorte, J. Chem. Phys. 93, 5337 (1990).

[11] W. T. Petrie and J. M. Vohs, J. Chem. Phys. 101, 8098 (1994).

[12] M. Haruta, Catal. Today 36, 153 (1997).

[13] E. J. Walter, S. P. Lewis, and A. M. Rappe, Surf. Sci. 495, 44 (2001).

[14] C. Bozo, N. Guilhaume, and J.-M. Herrmann, J. Catal. 393, 393 (2001).

[15] L. M. Molina and B. Hammer, Phys. Rev. Lett. 90, 206102 (2003).

[16] M. S. Chen and D. W. Goodman, Science 306, 252 (2004).

[17] X. She and M. Flytzani-Stephanopoulos, J. Catal. 237, 79 (2006).

[18] J. Feng, W. Zhang, and W. Jiang, Phys. Rev. B 72, 115423 (2005).

[19] D. R. Lide, ed., CRC Handbook of Chemistry and Physics, 85th Edition (CRC Press, Boca Raton, FL, 2004).

[20] J. P. Perdew, K. Burke, and M. Ernzerhof, Phys. Rev. Lett. 77, 3865 (1996).

[21] R. D. King-Smith and D. Vanderbilt, Phys. Rev. B 47, 1651 (1993).

[22] X. Gonze, D. C. Allan, and M. P. Teter, Phys. Rev. Lett.
68, 3603 (1992).

[23] H. J. Monkhorst and J. D. Pack, Phys. Rev. B 13, 5188 (1976)

[24] A. M. Rappe, K. M. Rabe, E. Kaxiras, and J. D. Joannopoulos, Phys. Rev. B Rapid Comm. 41, 1227 (1990).

[25] N. J. Ramer and A. M. Rappe, Phys. Rev. B 59, 12471 (1999).

[26] I. Grinberg, N. J. Ramer, and A. M. Rappe, Phys. Rev. B Rapid Comm. 63, 201102(R) (2001).

[27] http://opium.sourceforge.net.

[28] W. E. Lee and K. P. D. Lagerlof, J. Electron Microsc. Techn. 2, 247 (1985).

[29] C. Ruberto, Y. Yourdshahyan, and B. I. Lundqvist, Phys. Rev. B 67, 195412 (2003).

[30] Y. F. Zhukovskii, M. Alfredsson, K. Hermansson, E. Heifets, and E. A. Kotomin, Nucl. Instrum. Meth. Phys. Res. B 141, 73 (1998).

[31] Y. F. Zhukovskii, E. A. Kotomin, B. Herschend, K. Hermansson, and P. Jacobs, Surf. Sci. 513, 343 (2002).

[32] J. R. B. Gomes, Z. Lodziana, and F. Illas, J. Phys. Chem. B 107, 6411 (2003).

[33] I. D. Brown, in Structure and Bonding in Crystals II, edited by M. O'Keeffe and A. Navrotsky (Academic Press, New York, New York, 1981), pp. 1-30.

[34] A. Ditsch, P. E. Laibinis, D. I. C. Wang, and T. A. Hatton, Langmuir 21, 6006 (2005).

[35] X. Gonze and C. Lee, Phys. Rev. B 55, 10355 (1997).

[36] P. Ghosez, J.-P. Michenaud, and X. Gonze, Phys. Rev. B 58, 6224 (1998).

[37] B. Hammer, Y. Morikawa, and J. K. Nørskov, Phys. Rev. Lett. 76, 2141 (1996).

[38] M. Mavrikakis, B. Hammer, and J. K. Nørskov, Phys. Rev. Lett. 81, 2819 (1998).

[39] B. Hammer, O. H. Nielsen, and J. K. Nørskov, Catal. Lett. 46, 31 (1997).

[40] A. Ruban, B. Hammer, P. Stoltze, H. L. Skriver, and J. K. Nørskov, J. Mol. Catal. A: Chem. 115, 421 (1997).

[41] V. R. Cooper, A. M. Kolpak, Y. Yourdshahyan, and A. M. Rappe, Phys. Rev. B Rapid Comm. 72, 081409(R) (2005). 